\title{
The clinical significance of plasma clusterin and $A \beta$ in the longitudinal follow-up of patients with Alzheimer's disease
}

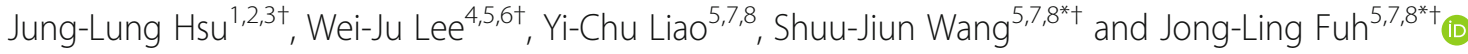

\begin{abstract}
Background: Clusterin and beta-amyloid $(A \beta)$ are involved in the pathogenesis of Alzheimer's disease (AD). The clinical significance of plasma clusterin and $A \beta$ in $A D$ progression remains controversial.

Methods: We recruited 322 patients with AD and 88 controls between August 2012 and June 2013. All participants were evaluated at baseline with a clinical assessment, Mini-Mental State Examination (MMSE), and Clinical Dementia Rating (CDR) scales. Patients with AD were evaluated annually with the MMSE and Neuropsychiatric Inventory (NPI) scale during the 2-year follow-up period. The levels of plasma clusterin, A $\beta 1-40$, and A $31-42$ at baseline were analyzed to study the longitudinal changes in the patient scores on the MMSE and NPI during the follow-up period.

Results: Patients in the highest tertile of plasma clusterin levels showed significantly lower MMSE scores than those in the lowest tertile $(p=0.04)$. After adjustment for multiple covariates using the generalized estimating equation analysis, there was a significant decrease in the MMSE scores over the 2-year follow-up period among AD patients in the highest tertile of plasma clusterin levels compared with those in the lowest tertile $(-2.09,95 \%$ confidence interval $(C l)=-3.67$ to $-0.51, p=0.01$ ). In apolipoprotein $E(A p o E) 4$-positive AD patients, baseline measurements of the ratio of plasma $A \beta 1-42 / A \beta 1-40$ in the highest tertile predicted an increase in NPI agitation/aggression scores over the 2-year follow-up period $(6.06,95 \% \mathrm{Cl}=1.20-10.62, p=0.02)$.
\end{abstract}

Conclusions: Plasma clusterin could serve as a biomarker for the severity of cognitive decline. Plasma $A \beta$ in ApoE4-positive AD could predict long-term agitation/aggression symptoms.

Keywords: Clusterin, Alzheimer's disease, Predictor, Progression

\section{Background}

Alzheimer's disease (AD) is the most common neurodegenerative dementia. It is characterized by a progressive loss of memory and other cognitive functions, and ultimately leads to total dependence. The early diagnosis and prediction of prognosis in $\mathrm{AD}$ is crucial but often very difficult in the early stages of the disease. In addition, $75-90 \%$ of $\mathrm{AD}$ patients develop "non-cognitive" behaviors, affect/mood, and psychosis symptoms that are collectively described as behaviors and psychological symptoms of dementia (BPSD). BPSD are classified into various clusters using the Neuropsychiatric Inventory (NPI) [1-3]. Two biomarkers,

\footnotetext{
* Correspondence: sjwang@vghtpe.gov.tw; jlfuh@vghtpe.gov.tw

${ }^{\dagger}$ Equal contributors

${ }^{5}$ Faculty of Medicine, National Yang-Ming University School of Medicine,

Taipei, Taiwan

Full list of author information is available at the end of the article
}

amyloid beta peptide $(\mathrm{A} \beta)$ and clusterin, are associated with AD-related phenotypes $[4,5]$. Previous reports have shown significant changes in the plasma levels of $A \beta$ in patients with $\mathrm{AD}[6,7]$, and a decrease in the plasma $A \beta 1-42 / A \beta 1-$ 40 ratio was inversely correlated with the neocortical amyloid burden, which is the key pathogenic process in $A D$. However, other studies have not detected an association between the plasma $\mathrm{A} \beta$ levels and $\mathrm{AD}$ [8]. This controversy about the relationship between the plasma $A \beta$ levels and $\mathrm{AD}$ may be the result of using different methods to measure $A \beta$ and other factors that may interfere with the detection of $A \beta$, such as albumin and transthyretin $[9,10]$. Longitudinal studies have reported both positive and negative associations between plasma $A \beta$ levels and cognitive decline, and the long-term clinical role of plasma $A \beta$ levels remains to be determined $[4,11]$. 
Clusterin, also known as apolipoprotein-J, is a multifunctional lipoprotein involved in $A \beta$ fibrillation, clearance, and complement inhibition [12, 13]. Clusterin is expressed in many tissues, and its expression in the brain is increased in $\mathrm{AD}[14,15]$. In $\mathrm{AD}$ patients, clusterin co-localizes with dystrophic neurites as well as $A \beta$ in amyloid plaques [16]. Studies of cerebrospinal fluid (CSF) have found both elevated and decreased levels of clusterin in $\mathrm{AD}$ patients $[17,18]$. It remains to be determined whether the CSF clusterin levels can be used for the diagnosis of $\mathrm{AD}$. More consistent data were derived from plasma studies. Elevated plasma clusterin levels in $\mathrm{AD}$ are correlated with $\mathrm{AD}$ pathology, prevalence, and severity; therefore, elevated clusterin levels have been suggested as a peripheral signature of AD [19-21]. One longitudinal study suggested that the plasma clusterin level was a good candidate marker for disease progression in AD [5]. Some studies of patients with mild cognitive impairment (MCI) or $\mathrm{AD}$ showed that elevated levels of plasma clusterin were associated with rapid cognitive decline, whereas other studies reported the reverse $[22,23]$.

Previous studies on prognostic factors of AD identified the age of onset, years of education, and disease severity at baseline as independent predictors of rapid cognitive decline $[24,25]$. While the plasma clusterin level may or may not predict cognitive changes in $\mathrm{AD}$, the clinical importance of plasma $A \beta$ levels over time remains unknown. In BPSD, early executive dysfunction may be a predictor of subsequent behavior disturbance [26] There is nothing known about the association between plasma biomarkers and BPSD. Plasma A $\beta$ may associate with clusterin as part of a clearance mechanism and contribute to other symptoms of AD over time [13]. In this study, we explored the clinical significance of plasma clusterin and $A \beta$ during a longitudinal study of patients with $\mathrm{AD}$.

\section{Methods}

\section{Participants}

We recruited patients with $\mathrm{AD}$ from the Neurology Department of Taipei Veterans General Hospital and the Taichung Veterans General Hospital in Taiwan between August 2012 and June 2014. Subjects who were between 60 and 90 years of age, of both genders, and had adequate visual and auditory abilities to perform all aspects of the study assessments were included. Diagnoses of $\mathrm{AD}$ were made by consensus during a multidisciplinary meeting based on the clinical criteria for probable $A D$ as described by the National Institute on Aging-Alzheimer's Association [27]. The exclusion criteria included any significant neurological disease other than AD that might affect cognition, including Parkinson's disease, multi-infarct dementia, Huntington's disease, normal pressure hydrocephalus, brain tumors, progressive supranuclear palsy, seizure disorders, subdural hematoma, multiple sclerosis, a history of significant head trauma followed by persistent neurologic defaults, or known structural brain abnormalities. The informant who received the examinations spent at least $10 \mathrm{~h}$ per week with the patient and was knowledgeable about their recent and past medical history. Controls were recruited from the spouses of $\mathrm{AD}$ patients included in the study and cognitively healthy patients at the Department of Neurology for the assessment of other disorders. Patients with a history of major neurological, psychiatric, or severe cardiovascular diseases were excluded. All participants received a standardized assessment that included a clinical interview, neuropsychological assessment, laboratory tests, and brain magnetic resonance imaging (MRI). Age, gender, body mass index (BMI), years of education, and disease duration were recorded for demographic analysis. Disease duration was defined as the onset of symptoms (as reported by the informant) until this study was performed. All information on patients and controls is summarized in Table 1. The Institutional Review Board of Taipei Veterans General Hospital approved this study (IRB number: 201205-033B). Written informed consent was obtained from all participants and their next of kin or legally authorized representatives.

\section{Clinical evaluation and procedures}

Cognitive function was assessed at baseline using standard procedures. The Mini-Mental State Examination (MMSE) was used to assess global cognition [28]. Clinical Dementia Rating (CDR) scores were used to represent the severity of dementia [29,30]. We also performed a memory test using the delayed recall of 12 items [31, 32], a modified Boston naming test [33], and a category verbal fluency test to assess short-term memory and language function [34]. Forward and backward digit-span tests were used to assess attention and working memory [35]. The NPI questionnaire was administered to assess the frequency and severity of BPSD [1, 2]. The NPI agitation/aggression, mood, and frontal symptoms subscales were used in this study [3]. The NPI agitation/aggression subscale includes agitation/aggression, disinhibition, irritability, and aberrant motor behavior items. The NPI mood subscale includes depression, anxiety, and irritability items. The NPI frontal subscale includes apathy, disinhibition, irritability, and euphoria items. Each subscale score was calculated by summing the scores for the severity of each item and then multiplying the summed score by the duration. Follow-up examinations for $\mathrm{AD}$ patients were performed at 1 and 2 years. At each follow-up, we re-evaluated patient cognition, BPSD, and disease severity using the MMSE, NPI, and CDR scores. All tests were administered by trained individuals in a clinical setting. 
Table 1 Demographic and clinical data of patients with AD and controls

\begin{tabular}{|c|c|c|c|c|c|}
\hline & $\begin{array}{l}\text { AD baseline } \\
(n=322)\end{array}$ & $\begin{array}{l}\text { AD 1-year follow-up } \\
(n=244)\end{array}$ & $\begin{array}{l}\text { AD 2-year follow-up } \\
(n=166)\end{array}$ & $\begin{array}{l}\text { Controls } \\
(n=88)\end{array}$ & $P$ value* \\
\hline Male patients, $n(\%)$ & $173(53.7 \%)$ & $128(52.4 \%)$ & $86(51.8)$ & $54(61.4 \%)$ & 0.19 \\
\hline Age (years) & $80.4(6.2)$ & $80.3(6.2)$ & $79.6(6.5)$ & $77.0(6.2)$ & $<0.01$ \\
\hline Education (years) & $10.1(4.4)$ & & & $10.1(5.1)$ & 0.96 \\
\hline Disease duration (months) & $52.4(47.5)$ & & & - & - \\
\hline BMI $\left(\mathrm{kg} / \mathrm{m}^{2}\right)$ & $23.6(3.4)$ & $23.7(3.3)$ & $23.9(3.3)$ & $24.4(3.2)$ & 0.07 \\
\hline MMSE & $18.1(5.9)$ & $16.7(6.4)$ & $16.5(6.6)$ & $27.2(2.5)$ & $<0.01$ \\
\hline Delayed recall of 12-item memory test & $1.1(1.8)$ & & & & \\
\hline Forward digit span & $8.5(3.2)$ & & & & \\
\hline Backward digit span & $3.9(2.1)$ & & & & \\
\hline Category verbal fluency & $6.1(3.0)$ & & & & \\
\hline Modified Boston naming test & $11.1(3.1)$ & & & & \\
\hline \multicolumn{6}{|l|}{ CDR, $n(\%)$} \\
\hline 0.5 & $20(6.2 \%)$ & $10(4.1 \%)$ & $6(3.6 \%)$ & & \\
\hline 1 & $208(64.6 \%)$ & $129(52.9 \%)$ & $74(44.6 \%)$ & & \\
\hline 2 & $81(25.2 \%)$ & $81(33.2 \%)$ & 65 (39.2\%) & & \\
\hline 3 & $12(3.7 \%)$ & $23(9.4 \%)$ & $19(11.4 \%)$ & & \\
\hline NPI-agitation/aggression & $7.0(12.0)$ & $7.9(12.5)$ & $6.9(11.4)$ & & \\
\hline NPI-mood & $5.4(9.4)$ & $6.4(9.9)$ & $5.3(8.6)$ & & \\
\hline NPI-frontal & $5.7(10.1)$ & $7.2(11.4)$ & $5.7(8.4)$ & & \\
\hline ApoE4 carrier, $n(\%)$ & $135(41.9 \%)$ & & & $9(10.2 \%)$ & $<0.01$ \\
\hline Plasma clusterin level ( $\mu \mathrm{g} / \mathrm{ml})$ & $248.6(37.9)$ & & & $243.8(35.9)$ & 0.29 \\
\hline $\mathrm{A} \beta 1-40(\mathrm{pg} / \mathrm{ml})$ & $157.9(50.7)$ & & & $144.5(36.9)$ & 0.02 \\
\hline $\mathrm{A} \beta 1-42(\mathrm{pg} / \mathrm{ml})$ & $36.9(20.8)$ & & & $34.1(9.1)$ & 0.23 \\
\hline Ratio of $A \beta 1-42 / A \beta 1-40(\%)$ & $23.7(8.7)$ & & & $24.4(6.7)$ & 0.32 \\
\hline
\end{tabular}

Values are shown as means and standard deviations unless otherwise indicated

*Comparison between AD baseline and controls by Independent two sample $t$ tests or Chi-square tests

$A \beta$ amyloid beta, $A D$ Alzheimer's disease, ApoE apolipoprotein E, BMI body mass index, CDR Clinical Dementia Rating, MMSE Mini-Mental State Examination, NPI Neuropsychiatric Inventory

\section{DNA and plasma clusterin level analyses}

Blood samples were collected in tubes containing sodium EDTA as an anticoagulant. Following centrifugation, plasma was aliquoted into polypropylene tubes and stored at $-80{ }^{\circ} \mathrm{C}$ until analysis. Genomic DNA was isolated from whole blood using the Gentra Puregene kit according to the manufacturer's protocols (Qiagen, Hilden, Germany). The presence of the $\varepsilon 2, \varepsilon 3$, and $\varepsilon 4$ alleles of the apolipoprotein gene (ApoE) was determined by assessing the sequences at two single-nucleotide polymorphisms (SNPs; rs429358 and rs7412) [36, 37]. An ApoE4 gene carrier was defined as having at least one allele containing the $\varepsilon 4$ gene. The plasma $A \beta$ peptide assay was performed using the INNO-BIA plasma $A \beta$ forms assay (Innogenetics, Ghent, Belgium), which is based on the multiplex xMAP technique, with a LABScan-100 system (Luminex BV, Netherlands). The ratio of plasma $A \beta 1-42 / A \beta 1-40$ was calculated as the plasma $A \beta 1-42$ level divided by the plasma $A \beta 1-40$ level. There was a significant difference in the ratio of plasma $A \beta 1-42 / A \beta 1-40$ between patients with $A D$ and controls [38]. Each sample was assessed in duplicate. The plasma clusterin levels were measured using a $\mathrm{Hu}$ man Clusterin Quantikine ELISA Kit (R\&D Systems, Minneapolis, MN, USA) according to the manufacturer's instructions. All samples from each participant were measured on the same plate to avoid interplate variation. The average intra-assay coefficient of variation based on duplicate samples was $1.2 \%$ for clusterin, $7.6 \%$ for $A \beta 1-$ 40 , and $9.1 \%$ for $A \beta 1-42$. The average inter-assay coefficient of variation, as determined using three samples (low, medium, and high measurement range) that were analyzed on 4 different days, was $3.3 \%$ for clusterin, $12.0 \%$ for $\mathrm{A} \beta 1-40$, and $15.8 \%$ for $\mathrm{A} \beta 1-42$.

\section{Statistical analysis}

All statistical analyses were performed using SPSS (version 21.0). Continuous variables are expressed as the means \pm 
standard deviations. Categorical variables are presented as numbers and ratios. Independent $t$ tests were performed to compare the ages, years of education, BMI, and MMSE scores of patients. Chi-square tests were used to compare $\mathrm{AD}$ patients and controls in terms of gender and ApoE4 carrier status. The analysis of variance (ANOVA) test and Kruskal-Wallis test were used to compare MMSE and NPI subscale scores with different levels of AD severity. We classified baseline plasma clusterin and $A \beta$ levels into tertiles (low, median, and high) to study mean changes in MMSE scores at the 1-year follow-up (MMSE at 1-year subtracted from MMSE at baseline). One-way ANOVA tests were used to compare group differences. To investigate the relationship between plasma biomarkers and MMSE and NPI subscale scores during longitudinal follow-up, the generalized estimating equation (GEE) method was used to analyze longitudinally correlated data. Baseline plasma clusterin levels and the plasma A $\beta 1-42$ / $\mathrm{A} \beta 1-40$ ratio in patients with $\mathrm{AD}$ were then stratified into tertiles (low, median, and high). Analyses were performed using the GEE method because it accounts for variations in time and identifies correlations among repeat measurements that are collected during a longitudinal study design. In addition, the MMSE and NPI subscale scores did not display a normal distribution. For the GEE analyses, we used a robust estimator for the covariance matrix and selected autoregressive (1) for the working correlation matrix. Age, years of education, gender, and ApoE4 gene status were used to adjust the MMSE and NPI subscale scores. Interactions between groups, time, and ApoE4 carrier status were also analyzed by GEE. Estimated $\beta$ values with 95\% confidence intervals (CIs) were calculated for all the blood biomarkers. Statistical significance was defined as $p<0.05$.

\section{Results}

\section{Demographic and clinical data}

In total, $322 \mathrm{AD}$ patients (173 males and 149 females; mean age $80.4 \pm 6.2$ years; mean education $10.1 \pm$ 4.4 years) and 88 controls (54 males and 34 females; mean age $=77.0 \pm 6.2$ years; mean education $=10.1 \pm$ 5.1 years) were recruited. Controls were significantly younger than patients in the $\mathrm{AD}$ group $(p<0.01)$. There were no significant differences between the groups in terms of gender or years of education. MMSE scores differed significantly between patients with AD and controls (Table $1 ; p<0.01$ ). Compared with controls, a significantly higher proportion patients with AD were ApoE4 carriers $(p<0.01)$. The plasma A $\beta 1-40$ levels were significantly higher in patients with $\mathrm{AD}$ compared with controls (Table $1 ; p=0.02$ ). The plasma $A \beta 1-42$ and clusterin levels were similar between groups $(p=0.23$ and 0.29 , respectively). To study the differences in the plasma $\mathrm{A} \beta$ and clusterin levels between patients with $\mathrm{AD}$ and controls, regression analyses were performed to adjust for the effects of age, gender, ApoE4 carrier status, and years of education. Our results showed no significant differences between groups in the plasma $A \beta 1-40$ or $A \beta 1-42$, the $\mathrm{A} \beta 1-42 / \mathrm{A} \beta 1-40$ ratio, or clusterin levels after correcting for multiple covariates $(p=0.15,0.17,0.69$, and 0.29 , respectively).

\section{Baseline characteristics for the complete study groups and the missing data group}

Ultimately, 244 AD patients (75.8\%) completed the 1-year clinical follow-up; 166 AD patients (51.5\%) completed the 2-year clinical follow-up. At the 1-year follow up, the baseline characteristics (age, gender, years of education, ApoE4 carrier status, MMSE scores, CDR stage, NPI subscale scores, plasma clusterin, and plasma A $\beta$ levels) were similar in both groups. At the 2-year follow-up, patients who did not complete the study were significantly older than those who participated in follow-ups (81.2 \pm 5.8 vs. $79.6 \pm 6.5$ years, $p=0.02$ ) and had lower MMSE scores $(17.2 \pm 6.1$ vs. $19.2 \pm 5.2, p<0.01)$. There was no significant difference in ApoE4 carrier status, CDR stage, NPI subscale scores, plasma clusterin level, or A $\beta$ level between the groups.

\section{Plasma biomarkers and dementia severity}

To clarify the relationship between plasma biomarkers and disease severity, we stratified patients based on the severity of $\mathrm{AD}$, as determined by the CDR scores. No significant differences in age, gender, or years of education were found among patients with different levels of AD severity. One-way ANOVA revealed no difference between the groups in terms of plasma clusterin, $A \beta 1-$ 42 levels, and the $\mathrm{A} \beta 1-42 / \mathrm{A} \beta 1-40$ ratio $(p=0.15,0.51$, and 0.08 , respectively). There was a significant difference between the groups in the plasma $A \beta 1-42$ levels (oneway ANOVA, $p<0.01$ ), and post-hoc analysis showed that the plasma $A \beta 1-42$ levels were significantly higher in the $\mathrm{CDR}=3$ group than in the $\mathrm{CDR}=1$ or 2 group $(p<0.05$; Table 2$)$.

\section{Baseline plasma biomarkers and ApoE4 carrier status in AD}

The Kruskal-Wallis test revealed that patients with severe $\mathrm{AD}$ were significantly more likely to carry the ApoE4 allele (Table $2 ; p<0.01$ ). Among the patients who were categorized as ApoE4 positive or negative, there was no significant difference in plasma levels of $A \beta 1-40$, $A \beta 1-42$, or the $A \beta 1-42 / A \beta 1-40$ ratio. However, the plasma clusterin levels were significantly higher in the ApoE4-positive group $(254.4 \pm 33.8 \mu \mathrm{g} / \mathrm{ml}$ vs. $244.4 \pm$ $40.2 \mu \mathrm{g} / \mathrm{ml}, p=0.02)$. ApoE4 carrier status was used as a covariate in the longitudinal follow-up study. 
Table 2 Demographic data of the patients with AD at baseline by disease severity

\begin{tabular}{|c|c|c|c|c|c|}
\hline & $\begin{array}{l}C D R=0.5 \\
(n=20)\end{array}$ & $\begin{array}{l}C D R=1.0 \\
(n=208)\end{array}$ & $\begin{array}{l}C D R=2.0 \\
(n=81)\end{array}$ & $\begin{array}{l}C D R=3.0 \\
(n=12)\end{array}$ & $P$ value* \\
\hline Male patients, $n(\%)$ & $13(65.0 \%)$ & $104(50.0 \%)$ & $49(60.5 \%)$ & $7(58.3 \%)$ & 0.29 \\
\hline Age (years) & $79.2(5.4)$ & $80.3(6.0)$ & $80.6(6.7)$ & $82.5(7.7)$ & 0.49 \\
\hline Education (years) & $9.6(4.9)$ & $10.2(4.5)$ & $9.7(4.3)$ & $12.4(3.6)$ & 0.22 \\
\hline Disease duration (months) & $24.8(16.7)$ & $51.1(48.6)$ & $56.4(39.7)$ & $99.3(71.6)$ & $<0.01$ \\
\hline $\mathrm{BMI}\left(\mathrm{kg} / \mathrm{m}^{2}\right)$ & $24.5(3.4)$ & $23.6(3.2)$ & $23.9(3.5)$ & $20.3(3.1)$ & 0.03 \\
\hline MMSE & $22.8(2.9)$ & $19.9(4.2)$ & $14.2(5.0)$ & $3.7(3.5)$ & $<0.01$ \\
\hline NPI-agitation/aggression & $5.5(13.3)$ & $5.5(10.0)$ & $10.1(13.5)$ & $14.8(22.1)$ & $<0.01$ \\
\hline NPI-mood & $6.3(12.9)$ & $4.7(8.6)$ & $6.5(8.8)$ & $9.8(16.2)$ & 0.15 \\
\hline NPI-frontal & $5.1(10.1)$ & $4.6(8.9)$ & $8.0(10.5)$ & $11.3(19.5)$ & 0.01 \\
\hline ApoE4 carrier & $6(30 \%)$ & 77 (37.0\%) & $44(54.3 \%)$ & $7(58.3 \%)$ & $<0.01$ \\
\hline Plasma clusterin level ( $\mu \mathrm{g} / \mathrm{ml})$ & $253.6(36.9)$ & $244.9(36.6)$ & $255.9(40.9)$ & $252.7(38.9)$ & 0.15 \\
\hline $\mathrm{A} \beta 1-40(\mathrm{pg} / \mathrm{ml})$ & $144.7(28.7)$ & $160.4(47.8)$ & $154.7(55.5)$ & $162.8(87.2)$ & 0.51 \\
\hline$A \beta 1-42(p g / m l)$ & $38.9(11.9)$ & $34.1(13.9)$ & $33.6(15.6)$ & $57.4(87.4)$ & $<0.01$ \\
\hline Ratio of $A \beta 1-42 / A \beta 1-40$ (\%) & $26.9(5.7)$ & $21.8(9.2)$ & $22.5(10.8)$ & $28.3(17.8)$ & 0.08 \\
\hline
\end{tabular}

Values are means with standard deviations unless otherwise indicated

*ANOVA or Kruskal-Wallis tests

$A \beta$ amyloid beta, $A D$ Alzheimer's disease, $A p o E$ apolipoprotein E, BMI body mass index, CDR Clinical Dementia Rating, MMSE Mini-Mental State Examination, NPI Neuropsychiatric Inventory

\section{Plasma clusterin and $A \beta$ levels at baseline and the 1-year follow-up}

Based on the baseline plasma clusterin levels, we stratified patients into high (mean $=289.4 \pm 22.6 \mu \mathrm{g} /$ $\mathrm{ml})$, medium $($ mean $=246.9 \pm 8.1 \mu \mathrm{g} / \mathrm{ml})$, and low (mean $=209.3 \pm 23.4 \mu \mathrm{g} / \mathrm{ml}$ ) tertiles to determine whether there was an association with baseline MMSE scores by one-way ANOVA. There was a significant group difference in MMSE scores (mean MMSE scores were $17.5 \pm 6.3$ in the high tertile, 18.1 \pm 5.6 in the medium tertile, and $19.4 \pm 4.6$ in the low tertile; $p=0.04$ ). Post-hoc analysis showed a difference between the high and low tertiles $(p<0.05)$. We further studied mean changes in MMSE scores at the 1year follow-up. A significant within-group difference was found by one-way ANOVA (mean changes in MMSE scores at 1 year were $-2.5 \pm 3.4$ in the high tertile, $-2.0 \pm 4.5$ in the medium tertile, and $-0.9 \pm 3.5$ in the low tertile; $p=0.02$ ). Post-hoc analysis showed a significant difference between the high and low tertiles $(p=0.008)$. This suggests an association between higher plasma clusterin levels and lower cognitive status in patients with $\mathrm{AD}$. For the analysis of plasma $\mathrm{A} \beta, \mathrm{A} \beta 1-40, \mathrm{~A} \beta 1-42$, and the $\mathrm{A} \beta 1-42 / \mathrm{A} \beta 1-40$ ratio, patients were stratified into tertiles and we compared the mean changes in the MMSE scores at the 1-year follow-up using a one-way ANOVA. There were no significant differences in mean changes in MMSE scores among the tertiles of $A \beta 1-40, A \beta 1-42$, or the $\mathrm{A} \beta 1-42 / \mathrm{A} \beta 1-40$ ratio $(p=0.77,0.15$, and 0.43 , respectively).

\section{Plasma clusterin levels at the 2-year follow-up}

Longitudinal data collected over 2 years were analyzed by GEE adjusted for age, years of education, gender, time, and ApoE4 carrier status. This revealed that the MMSE scores were significantly decreased in patients in the high tertile of plasma clusterin levels (average decrease in MMSE scores after 2 years was -2.09 points, 95\% CI $=-3.67$ to $-0.51, p=0.01)$ compared with patients in the low tertile (Fig. 1). This MMSE difference was the same difference as observed at baseline. No significant interactions were detected between the tertiles of plasma clusterin and time or between ApoE4 status and time $(p=0.10$ and $p=0.16$, respectively). MMSE scores were significantly lower among patients carrying the ApoE4 allele than those without $(-2.75,95 \% \mathrm{CI}=$ -4.11 to $-1.39, p<0.01)$. Because plasma clusterin was significantly higher in the ApoE4-positive group, we performed an interaction analysis. No significant interaction between clusterin tertile and ApoE4 carrier status was detected ( $p=0.93$; Table 3$)$. The plasma clusterin levels showed no association with agitation/aggression, mood, or frontal subscale NPI scores.

\section{Longitudinal analysis of plasma $A \beta$}

To explore the predictive value of plasma $A \beta$ for cognition and BPSD, we stratified patients by the plasma $\mathrm{A} \beta 1-42 / \mathrm{A} \beta 1-40$ ratio into high (mean $=31.9 \pm 10.2 \%$ ), medium $($ mean $=22.6 \pm 1.4 \%)$, or low $\quad($ mean $=16.7 \pm$ $2.5 \%)$ groups. After adjustment for age, years of education, gender, time, and ApoE4 carrier status, GEE analysis revealed no association between the plasma $A \beta 1-$ 


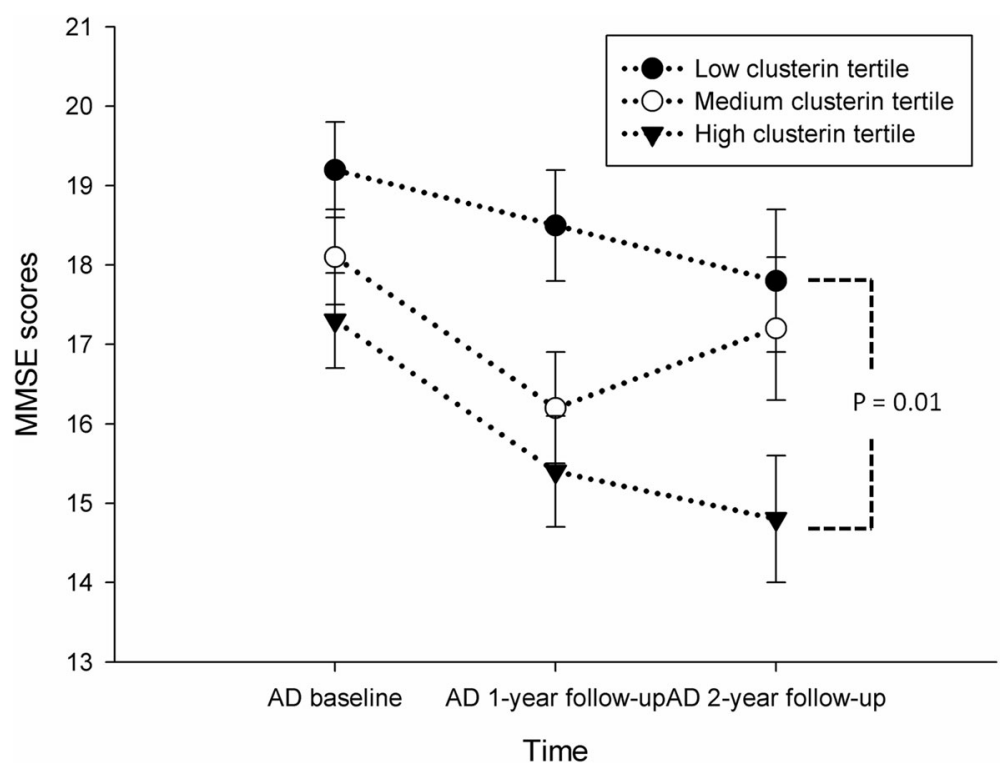

Fig. 1 Plasma biomarkers associated with longitudinal changes in MMSE scores. Patients in the high tertile of plasma clusterin levels had significantly lower Mini-Mental State Examination (MMSE) scores compared with those in the low tertile based on a GEE analysis adjusted for age, gender, years of education, and ApoE4 carrier status $(p=0.01)$. There was no significant interaction between time and the tertile of plasma clusterin $(p=0.10)$. Data are presented as the mean \pm standard error. AD Alzheimer's disease

42/A $\beta 1-40$ ratio and the decline in the MMSE score at 2 years $(p=0.12)$. GEE analysis showed that the plasma $\mathrm{A} \beta 1-42 / \mathrm{A} \beta 1-40$ ratio and ApoE4 carrier status were significant predictors of the NPI agitation/aggression score $(p=0.04$ and 0.009 , respectively). GEE analysis also revealed a significant interaction between the plasma $A \beta 1-$ 42/A $\beta 1-40$ ratio and ApoE4 carrier status $(p=0.003)$.

Table 3 Results of the generalized estimating equation analyzing the effect of 2-year MMSE changes in patients with AD

\begin{tabular}{lllll}
\hline & $\beta$ & SE & $95 \% \mathrm{Cl}$ & $P$ value \\
\hline $\begin{array}{lllll}\text { Gender } \\
\text { Male }\end{array}$ & -0.32 & 0.83 & $(-1.95,1.32)$ & 0.71 \\
$\quad$ Female & Referent & & & \\
$\begin{array}{l}\text { Clusterin level } \\
\text { High }\end{array}$ & -2.09 & 0.81 & $(-3.67,-0.51)$ & 0.01 \\
$\quad$ Medium & -0.87 & 0.71 & $(-2.27,0.53)$ & 0.22 \\
Low & Referent & & & \\
ApoE4 carrier & & & & \\
$\quad$ Positive & -2.75 & 0.69 & $(-4.11,-1.39)$ & 0.00 \\
$\quad$ Negative & Referent & & & \\
Age & 0.03 & 0.08 & $(-0.12,0.17)$ & 0.74 \\
Education years & 0.13 & 0.09 & $(-0.04,0.30)$ & 0.15 \\
Time & -0.91 & 0.21 & $(-1.33,-0.49)$ & 0.00 \\
\hline Adjusted for age, eduction yars & & & & \\
\hline
\end{tabular}

Adjusted for age, education years, gender, time, and ApoE4 carrier Interaction in group ${ }^{*}$ ApoE4 carrier: $p$ value $=0.93$ and group $*$ time: $p$ value $=0.21$

$A D$ Alzheimer's disease, ApoE apolipoprotein $\mathrm{E}, \mathrm{Cl}$ confidence interval, MMSE Mini-Mental State Examination, SE standard error
Next, we stratified patients with AD based on ApoE4 carrier status (135 ApoE4 carriers and 187 non-carriers). After 2 years, ApoE4 carriers in the high plasma A $\beta 1-42 /$ A $\beta 1-40$ ratio tertile showed a significant increase in the NPI agitation/aggression score compared with those in the low tertile $(6.06,95 \% \mathrm{CI}=1.20-10.62, p=0.02$; Table 4 , Fig. 2a). No such association was found among ApoE4negative patients (Fig. $2 b$ ). The plasma $A \beta 1-42 / A \beta 1-40$ ratio was not a significant predictor of NPI-mood or NPIfrontal scores. GEE analysis revealed no significant association between plasma A $\beta 1-42$ level and MMSE or NPI subscale scores at 2 years.

\section{Discussion}

This study investigated the utility of plasma clusterin and $A \beta$ levels as prognostic markers in AD. Higher plasma clusterin levels were associated with significantly lower MMSE scores at baseline. At the 1- and 2-year follow-up, GEE analyses of AD patients revealed that high levels of plasma clusterin at baseline were associated with a significantly larger decrease in MMSE compared with low levels of plasma clusterin (after adjustment for multiple covariate factors). However, there was no significant interaction between time and the clusterin level tertile, which indicates that higher levels of plasma clusterin were not associated with the rapid decline of MMSE scores over time. The ApoE4 carrier status of patients with AD also displayed a similar pattern. Literature shows that ApoE4 and plasma clusterin are biomarkers for an accelerated cognitive 
Table 4 Results of the generalized estimating equation analyzing the effect of 2-year NPI agitation/aggression changes in ApoE4positive patients with $A D$

\begin{tabular}{lllll}
\hline & $\beta$ & SE & $95 \% \mathrm{Cl}$ & $P$ value \\
\hline $\begin{array}{l}\text { Gender } \\
\text { Male }\end{array}$ & 0.16 & 2.25 & $(-4.25,4.57)$ & 0.94 \\
$\begin{array}{l}\text { Female } \\
\text { Ratio of A } 31-42 / A \beta 1-40\end{array}$ & Ref & & & \\
$\quad$ High & 6.06 & 2.48 & $(1.20,10.62)$ & 0.02 \\
$\quad$ Medium & 1.20 & 2.24 & $(-3.18,5.59)$ & 0.59 \\
Low & Ref & & & \\
Age & 0.18 & 0.14 & $(-0.10,0.46)$ & 0.21 \\
Education years & 0.11 & 0.17 & $(-0.23,0.45)$ & 0.51 \\
Time & -0.96 & 0.70 & $(-2.34,0.42)$ & 0.17 \\
\hline Adust
\end{tabular}

Adjusted for age, education years, gender, and time

$A \beta$ amyloid beta peptide, $A D$ Alzheimer's disease, $\mathrm{Cl}$ confidence interval, NPI Neuropsychiatric Inventory, SE standard error

decline in AD [39]. However, our results indicate that the plasma clusterin level is a biomarker for the severity of cognitive function decline in patients with $\mathrm{AD}$ rather than a prognostic marker.

The results of our longitudinal study on plasma $A \beta$ levels showed no association with a change in cognition, which is consistent with previous findings $[4,40]$. In the ApoE4-positive group, patients in the high but not the low tertile for plasma $A \beta 1-42 / A \beta 1-40$ ratio showed a significant increase in NPI agitation/aggression scores at the 2-year follow-up. These findings indicate that the plasma $A \beta 1-42 / A \beta 1-40$ ratio could serve as a biomarker for agitation/aggression symptoms during the long-term follow-up of ApoE4 carriers. To the best of our knowledge, this has not been proposed in previous studies.

\section{Plasma clusterin levels and cognitive decline}

At present there is no reliable biomarker for predicting the speed of AD progression [41, 42]. The plasma level of clusterin could be a prognostic marker for patients with $\mathrm{AD}$, but the specific relationship between the clusterin level and cognitive decline remains to be determined. A previous study reported that higher concentrations of plasma clusterin were significantly associated with an accelerated cognitive decline in patients with AD after 1 year of follow-up [23]. In patients with $\mathrm{MCI}$, higher baseline levels of plasma clusterin have been associated with lower rates of brain atrophy. The authors of that study proposed that increased clusterin levels could protect at-risk subjects; failure of this mechanism in patients with $\mathrm{AD}$ would account for a more rapid decline in cognition and brain structural changes [43]. Another study reported conflicting results, showing that higher clusterin levels were related to less cognitive decline in patients with $\mathrm{AD}$ [22]. The results from our 2-year follow-up showed that higher levels of plasma clusterin were not associated with a more rapid cognitive decline in $\mathrm{AD}$, which indicates that plasma clusterin levels are related to cognitive state and disease severity in $\operatorname{AD}[19,44]$.

Many confounding factors may contribute to plasma clusterin levels. Recent studies have shown that plasma clusterin levels can be affected by gender, obesity, systemic inflammation, or atherogenic components of the lipid profile in AD patients $[45,46]$. Plasma clusterin levels also play a role in tissue remodeling and immune responses in the brain, which may be related to the speed of deterioration in AD patients [45-48]. Further research is necessary to explore a potential role for clusterin in response to tissue damage and how it could influence the progression of $\mathrm{AD}$. In practice, interpreting the plasma clusterin level is required to determine the disease state of a subject.

\section{Plasma $A \beta$ predicts agitation/aggression symptoms in ApoE4 carriers}

In our current work, we found that the plasma $A \beta 1-42 /$ A $\beta 1-40$ ratio at baseline could predict the increase in NPI agitation/aggression scores among ApoE4 carriers. Agitation and aggression symptoms are difficult to manage and are especially distressing for patients, caregivers, and medical staff members. In a previous study, the CSF level of $A \beta 1-42$ was associated with agitation and irritability symptoms in patients with MCI [49]. $\beta$-Amyloid precursor protein, a precursor for $A \beta$, has also been linked to aggression symptoms in young children with autism [50]. The ApoE4 gene also contributes to aggression symptoms in patients with $\mathrm{AD}[51,52]$. Our findings suggest that plasma $A \beta$ may act synergistically with the ApoE4 gene to aggravate agitation/aggression symptoms in patients with $\mathrm{AD}$ because the ApoE4-negative group did not show this association. Additional large, cohort studies with longitudinal follow-ups will be needed to confirm this finding.

\section{Plasma $A \beta$ and cognitive changes in $A D$}

Our study found no significant differences in the plasma $A \beta$ levels between patients with $A D$ and controls after adjustment for multiple covariates, nor was there a longitudinal association with MMSE changes in patients with $\mathrm{AD}$. Previous studies have shown increased plasma $\mathrm{A} \beta 1-40$ or $\mathrm{A} \beta 1-42$ levels in patients with $\mathrm{AD}[7,53]$, but negative studies have also been reported [54, 55]. Longitudinal studies on the association between the plasma $A \beta$ levels and cognitive changes have also shown both positive $[11,56]$ and negative results [4]. This variation in studies on plasma $A \beta$ may be the result of different collection methods [57], assay techniques [58], 

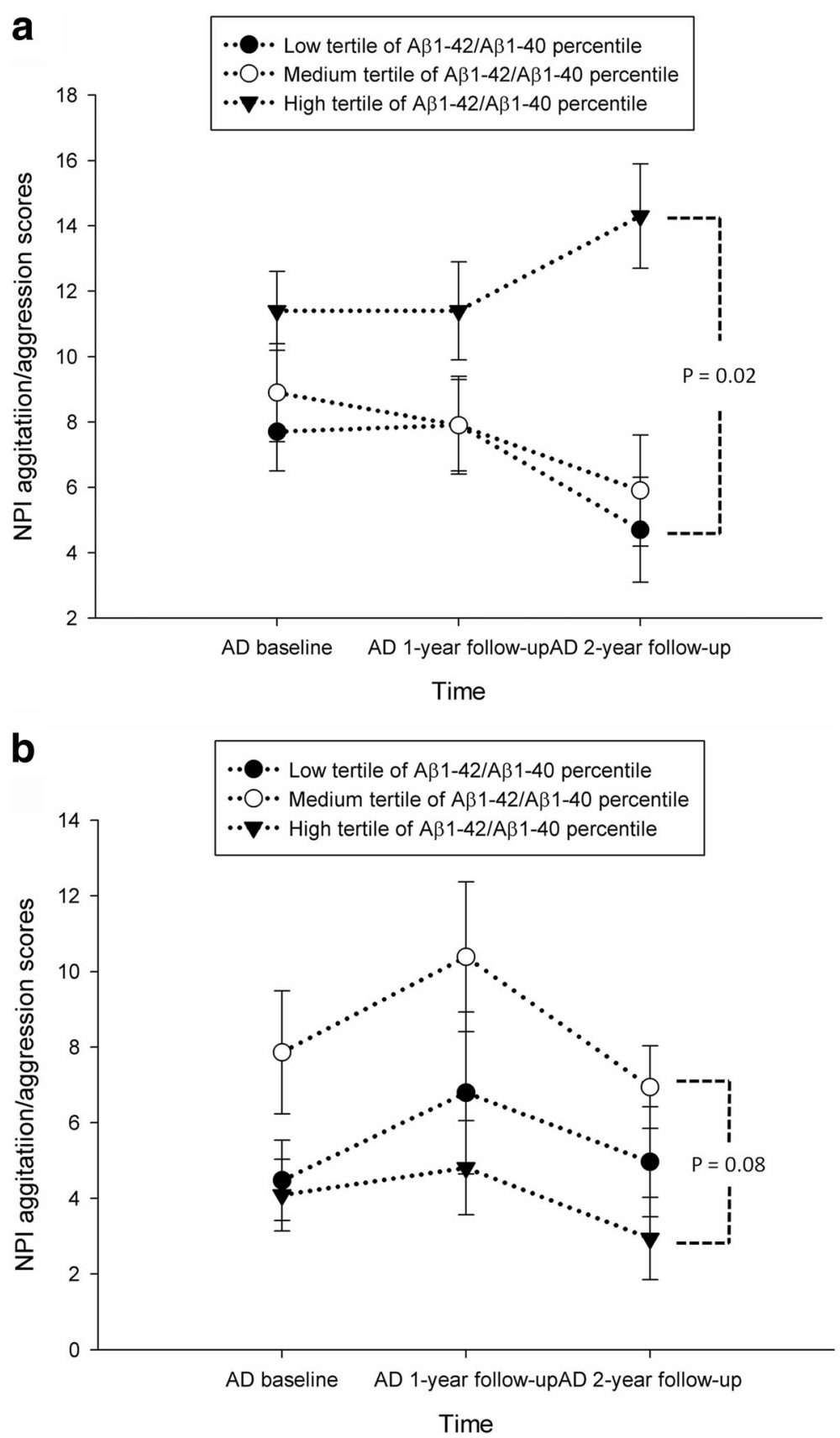

Fig. 2 Plasma biomarkers associated with longitudinal changes in NPI-agitation/aggression scores based on the differences in ApoE4 carrier status. a Compared with the $A \beta 1-42 / A \beta 1-40$ ratios in the low tertile, those in the high tertile were associated with a significant increase in $\mathrm{NPI}$-agitation/aggression scores in ApoE4-positive carriers based on a GEE analysis adjusted for age, gender, and years of education ( $p=0.02$ ). b There was no significant difference between groups for NPI-agitation/aggression scores in ApoE4-negative carriers based on a GEE analysis adjusted for age, gender, and years of education. Data are presented as the mean \pm standard error. $A \beta$ amyloid beta peptide, AD Alzheimer's disease, NPI Neuropsychiatric Inventory

and multiple confounding factors such as diabetes, hypertension, or medication use [59,60]. A recent study demonstrated an association between chemically treated plasma $A \beta$ and the deposition of brain amyloid, which may provide a potential blood-based biomarker for the study of AD [61].

\section{Limitations}

There are several limitations to the current study. Over the 2 years of follow-up, patients exhibited disease progression, cognitive decline, and an increased severity of neuropsychiatric symptoms, such as agitation and aggression [62]. This could be part of the natural course of 
the disease; MMSE and NPI symptoms associated with AD decline at different rates depending on the severity of $\mathrm{AD}$. Although levels of plasma clusterin did not differ significantly among patients with different levels of AD severity, we cannot exclude such a possibility. Other confounding factors, such as depression and agitation symptoms, have been reported to increase the plasma clusterin levels, and the plasma amyloid levels may be affected by genetic variants and creatinine levels $[47,63,64]$. We acknowledge this limitation, and consider both plasma biomarkers to be predictors of clinical phenotype, rather than exhibiting a direct causal relationship. Second, we did not include other plasma biomarkers or imaging data in this study. CSF A $31-42$ levels or brain amyloid scans could provide more reliable pathological markers than plasma biomarkers for the diagnosis of $\mathrm{AD}$ [65]. Measurements of structural changes in brain regions could be a robust marker, although it is more expensive than plasma biomarkers. Our previous cross-sectional study showed that the score for right-side posterior atrophy, as measured by brain MRI, was associated with NPI agitation/aggression symptoms [66]. A longitudinally repeated MRI measurement could be useful for linking plasma biomarkers with brain morphology and BPSD. Third, our drop-out rate at the 2-year follow-up was $49 \%$, and the missing data group had an older age and lower MMSE scores than did the complete study group. Although there were no differences in plasma clusterin or $A \beta$ levels between groups, we cannot exclude the possibility of bias caused by our missing data. A study with a larger sample size and a higher follow-up rate may improve our findings.

\section{Conclusions}

In summary, our results suggest that the highest tertile of baseline plasma clusterin levels are associated with lower MMSE scores. The plasma clusterin level could be a marker of cognitive severity rather than a prognostic marker in patients with $\mathrm{AD}$. A high plasma $\mathrm{A} \beta 1-42 /$ A $\beta 1-40$ ratio combined with the ApoE4 gene may increase NPI agitation/aggression symptoms in patients with AD after 2 years of follow-up, which indicates a surrogate prognostic marker. These results will provide clinicians and caregivers with information on disease severity and progression in patients with $\mathrm{AD}$, and give them a clear picture of the future to assist with designing a suitable care plan for patients with AD.

\section{Abbreviations}

AD: Alzheimer's disease; ANOVA: Analysis of variance; ApoE: Apolipoprotein E; Aß: Beta-amyloid; BMI: Body mass index; BPSD: Behaviors and psychological symptoms of dementia; CDR: Clinical Dementia Rating; Cl: Confidence interval; CSF: Cerebrospinal fluid; GEE: Generalized estimating equation; $\mathrm{MCl}$ : Mild cognitive impairment; MMSE: Mini-Mental State Examination; MRI: Magnetic resonance imaging; NPI: Neuropsychiatric Inventory

\section{Funding}

The study was supported by grants from the Academia Sinica of Taiwan (Taiwan Biobank: Biosignature Study of Alzheimer Disease), the Ministry of Science and Technology of Taiwan (104-2314-B-075-005-MY2, 104-2745-B-075-001, 106-2321B-075-001), Taipei Veterans General Hospital (V106C-126, V105D9-001-MY2-2, V106E-006-1), the Brain Research Center at National Yang-Ming University, and the Ministry of Education Aim for the Top University Plan. The funders had no role in the study design, data collection, data analysis, decision to publish, or preparation of the manuscript.

Availability of data and materials

Raw data will not be available due to patient confidentiality requirements.

\section{Authors' contributions}

JLH was the physician who generated the idea, conceived of the study design, and drafted the manuscript. WJL, YCL, and JLF contributed to case recruitment, study preparation, the interpretation of the results, and manuscript preparation. SJW and JLF were involved in grant applications and manuscript revision. All authors read and approved the final manuscript.

\section{Authors' information}

Not applicable.

\section{Ethics approval and consent to participate}

The Institutional Review Board of Taipei Veterans General Hospital approved this study. The IRB number is 2012-05-033B. Written informed consent was obtained from all participants and their next of kin or legally authorized representatives.

\section{Consent for publication}

Not applicable.

\section{Competing interests}

The authors declare that they have no competing interests.

\section{Publisher's Note}

Springer Nature remains neutral with regard to jurisdictional claims in published maps and institutional affiliations.

\section{Author details}

${ }^{1}$ Graduate Institute of Humanities in Medicine, Taipei Medical University, Taipei, Taiwan. ${ }^{2}$ Department of Neurology, Chang Gung Memorial Hospital Linkou Medical Center and College of Medicine, Chang-Gung University, Taoyuan, Taiwan. ${ }^{3}$ Taipei Medical University Research Center for Brain and Consciousness, Shuang-Ho Hospital, New Taipei City, Taiwan. ${ }^{4}$ Neurological Institute, Taichung Veterans General Hospital, Taichung, Taiwan. ${ }^{5}$ Faculty of Medicine, National Yang-Ming University School of Medicine, Taipei, Taiwan. ${ }^{6}$ Institute of Clinical Medicine, National Yang-Ming University School of Medicine, Taipei, Taiwan. ${ }^{7}$ Brain Research Center, National Yang-Ming University School of Medicine, Taipei, Taiwan. ${ }^{8}$ Department of Neurology, Neurological Institute, Taipei Veterans General Hospital, Taipei 112, Taiwan.

Received: 12 April 2017 Accepted: 6 November 2017

Published online: 23 November 2017

\section{References}

1. Cummings JL, Mega M, Gray K, Rosenberg-Thompson S, Carusi DA, Gornbein J. The Neuropsychiatric Inventory: comprehensive assessment of psychopathology in dementia. Neurology. 1994:44:2308-14.

2. Fuh JL, Wang SJ, Cummings JL. Neuropsychiatric profiles in patients with Alzheimer's disease and vascular dementia. J Neurol Neurosurg Psychiatry. 2005;76:1337-41.

3. Trzepacz PT, Saykin A, Yu P, Bhamditipati P, Sun J, Dennehy EB, et al. Subscale validation of the neuropsychiatric inventory questionnaire: comparison of Alzheimer's disease neuroimaging initiative and national Alzheimer's coordinating center cohorts. Am J Geriatr Psychiatry. 2013;21:607-22.

4. Donohue MC, Moghadam SH, Roe AD, Sun CK, Edland SD, Thomas RG, et al. Longitudinal plasma amyloid beta in Alzheimer's disease clinical trials. Alzheimers Dement. 2015;11:1069-79.

5. Kiddle SJ, Sattlecker M, Proitsi P, Simmons A, Westman E, Bazenet C, et al. Candidate blood proteome markers of Alzheimer's disease onset and 
progression: a systematic review and replication study. J Alzheimers Dis. 2014;38:515-31.

6. Kleinschmidt M, Schoenfeld R, Gottlich C, Bittner D, Metzner JE, Leplow B, et al. Characterizing aging, mild cognitive impairment, and dementia with bloodbased biomarkers and neuropsychology. J Alzheimers Dis. 2015;50:111-26.

7. Kim HJ, Park KW, Kim TE, Im JY, Shin HS, Kim S, et al. Elevation of the plasma Abeta40/Abeta42 ratio as a diagnostic marker of sporadic earlyonset Alzheimer's disease. J Alzheimers Dis. 2015;48:1043-50.

8. Fukumoto $\mathrm{H}$, Tennis M, Locascio JJ, Hyman BT, Growdon JH, Irizarry MC. Age but not diagnosis is the main predictor of plasma amyloid beta-protein levels. Arch Neurol. 2003;60:958-64.

9. Han SH, Jung ES, Sohn JH, Hong HJ, Hong HS, Kim JW, et al. Human serum transthyretin levels correlate inversely with Alzheimer's disease. J Alzheimers Dis. 2011;25:77-84.

10. Kuo YM, Kokjohn TA, Kalback W, Luehrs D, Galasko DR, Chevallier N, et al. Amyloid-beta peptides interact with plasma proteins and erythrocytes: implications for their quantitation in plasma. Biochem Biophys Res Commun. 2000;268:750-6.

11. Cosentino SA, Stern Y, Sokolov E, Scarmeas N, Manly JJ, Tang MX, et al. Plasma ss-amyloid and cognitive decline. Arch Neurol. 2010;67:1485-90.

12. Murphy BF, Saunders JR, O'Bryan MK, Kirszbaum L, Walker ID, d'Apice AJ. SP-40,40 is an inhibitor of C5b-6-initiated haemolysis. Int Immunol. 1989;1:551-4.

13. Nuutinen T, Suuronen T, Kauppinen A, Salminen A. Clusterin: a forgotten player in Alzheimer's disease. Brain Res Rev. 2009;61:89-104.

14. Harold D, Abraham R, Hollingworth P, Sims R, Gerrish A, Hamshere ML, et al. Genome-wide association study identifies variants at CLU and PICALM associated with Alzheimer's disease. Nat Genet. 2009;41:1088-93.

15. Lambert JC, Heath S, Even G, Campion D, Sleegers K, Hiltunen M, et al. Genome-wide association study identifies variants at CLU and CR1 associated with Alzheimer's disease. Nat Genet. 2009:41:1094-9.

16. Nielsen HM, Mulder SD, Belien JA, Musters RJ, Eikelenboom P, Veerhuis R. Astrocytic Abeta 1-42 uptake is determined by Abeta-aggregation state and the presence of amyloid-associated proteins. Glia. 2010;58:1235-46.

17. Sihlbom C, Davidsson P, Sjogren M, Wahlund LO, Nilsson CL. Structural and quantitative comparison of cerebrospinal fluid glycoproteins in Alzheimer's disease patients and healthy individuals. Neurochem Res. 2008;33:1332-40.

18. Puchades M, Hansson SF, Nilsson CL, Andreasen N, Blennow K, Davidsson P. Proteomic studies of potential cerebrospinal fluid protein markers for Alzheimer's disease. Brain Res Mol Brain Res. 2003;118:140-6.

19. Schrijvers EM, Koudstaal PJ, Hofman A, Breteler MM. Plasma clusterin and the risk of Alzheimer disease. JAMA. 2011;305:1322-6.

20. Gupta VB, Doecke JD, Hone E, Pedrini S, Laws SM, Thambisetty M, et al. Plasma apolipoprotein J as a potential biomarker for Alzheimer's disease: Australian Imaging, Biomarkers and Lifestyle study of aging. Alzheimers Dement (Amst). 2016:3:18-26.

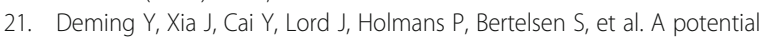
endophenotype for Alzheimer's disease: cerebrospinal fluid clusterin. Neurobiol Aging. 2016;37:208 e1-9.

22. Jongbloed W, van Dijk KD, Mulder SD, van de Berg WD, Blankenstein MA, van der Flier W, et al. Clusterin levels in plasma predict cognitive decline and progression to Alzheimer's disease. J Alzheimers Dis. 2015;46:1103-10.

23. Thambisetty M, Simmons A, Velayudhan L, Hye A, Campbell J, Zhang Y, et al. Association of plasma clusterin concentration with severity, pathology, and progression in Alzheimer disease. Arch Gen Psychiatry. 2010;67:739-48.

24. Jutkowitz E, MacLehose RF, Gaugler JE, Dowd B, Kuntz KM, Kane RL. Risk Factors Associated With Cognitive, Functional, and Behavioral Trajectories of Newly Diagnosed Dementia Patients. J Gerontol A Biol Sci Med Sci. 2017;72:251-8.

25. Lopez OL, Schwam E, Cummings J, Gauthier S, Jones R, Wilkinson D, et al. Predicting cognitive decline in Alzheimer's disease: an integrated analysis. Alzheimers Dement. 2010;6:431-9.

26. Tsoi T, Baillon S, Lindesay J. Early frontal executive impairment as a predictor of subsequent behavior disturbance in dementia. Am J Geriatr Psychiatry. 2008;16:102-8.

27. McKhann GM, Knopman DS, Chertkow H, Hyman BT, Jack Jr CR, Kawas CH, et al. The diagnosis of dementia due to Alzheimer's disease: recommendations from the National Institute on Aging-Alzheimer's Association workgroups on diagnostic guidelines for Alzheimer's disease. Alzheimers Dement. 2011;7:263-9.

28. Folstein MF, Folstein SE, McHugh PR. "Mini-mental state". A practical method for grading the cognitive state of patients for the clinician. J Psychiatr Res. 1975;12:189-98.
29. Hughes CP, Berg L, Danziger WL, Coben LA, Martin RL. A new clinical scale for the staging of dementia. Br J Psychiatry. 1982;140:566-72.

30. Burke WJ, Miller JP, Rubin EH, Morris JC, Coben LA, Duchek J, et al. Reliability of the Washington University Clinical Dementia Rating. Arch Neurol. 1988;45:31-2.

31. Vakil E, Blachstein H. Rey Auditory-Verbal Learning Test: structure analysis. J Clin Psychol. 1993;49:883-90.

32. Vanderploeg RD, Schinka JA, Jones T, Small BJ, Graves AB, Mortimer JA. Elderly norms for the Hopkins Verbal Learning Test-Revised. Clin Neuropsychol. 2000;14:318-24.

33. Mack WJ, Freed DM, Williams BW, Henderson WW. Boston Naming Test: shortened versions for use in Alzheimer's disease. J Gerontol. 1992;47: P154-8.

34. Barr A, Brandt J. Word-list generation deficits in dementia. J Clin Exp Neuropsychol. 1996;18:810-22.

35. Wechsler D. Wechsler Adult Intelligence Scale. 3rd ed. San Antonio, TX: The Psychological Corporation; 1997.

36. Chen CS, Ouyang P, Yeh YC, Lai CL, Liu CK, Yen CF, et al. Apolipoprotein E polymorphism and behavioral and psychological symptoms of dementia in patients with Alzheimer disease. Alzheimer Dis Assoc Disord. 2012;26:135-9.

37. Liao YC, Lee WJ, Hwang JP, Wang YF, Tsai CF, Wang PN, et al. ABCA7 gene and the risk of Alzheimer's disease in Han Chinese in Taiwan. Neurobiol Aging. 2014;35:2423 e7-23 e13.

38. Lewczuk P, Kornhuber J, Vanmechelen E, Peters O, Heuser I, Maier W, et al. Amyloid beta peptides in plasma in early diagnosis of Alzheimer's disease: a multicenter study with multiplexing. Exp Neurol. 2010;223:366-70.

39. De Beaumont L, Pelleieux S, Lamarre-Theroux L, Dea D, Poirier J, Alzheimer's Disease Cooperative S. Butyrylcholinesterase K and apolipoprotein Evarepsilon4 reduce the age of onset of Alzheimer's disease, accelerate cognitive decline, and modulate donepezil response in mild cognitively impaired subjects. J Alzheimers Dis. 2016;54:913-22.

40. Rembach A, Faux NG, Watt AD, Pertile KK, Rumble RL, Trounson BO, et al. Changes in plasma amyloid beta in a longitudinal study of aging and Alzheimer's disease. Alzheimers Dement. 2014;10:53-61.

41. Herrmann N, Harimoto T, Balshaw R, Lanctot KL, Canadian Outcomes Study in Dementia Investigators. Risk factors for progression of Alzheimer disease in a Canadian population: the Canadian Outcomes Study in Dementia (COSID). Can J Psychiatry. 2015;60:189-99.

42. Tschanz JT, Corcoran CD, Schwartz S, Treiber K, Green RC, Norton MC, et al. Progression of cognitive, functional, and neuropsychiatric symptom domains in a population cohort with Alzheimer dementia: the Cache County Dementia Progression study. Am J Geriatr Psychiatry. 2011;19:532-42.

43. Thambisetty M, An Y, Kinsey A, Koka D, Saleem M, Guntert A, et al. Plasma clusterin concentration is associated with longitudinal brain atrophy in mild cognitive impairment. Neuroimage. 2012;59:212-7.

44. Song F, Poljak A, Crawford J, Kochan NA, Wen W, Cameron B, et al. Plasma apolipoprotein levels are associated with cognitive status and decline in a community cohort of older individuals. PLoS One. 2012;7, e34078.

45. Baralla A, Sotgiu E, Deiana M, Pasella S, Pinna S, Mannu A, et al. Plasma clusterin and lipid profile: a link with aging and cardiovascular diseases in a population with a consistent number of centenarians. PLoS One. 2015;10, e0128029.

46. Won JC, Park CY, Oh SW, Lee ES, Youn BS, Kim MS. Plasma clusterin (ApoJ) levels are associated with adiposity and systemic inflammation. PLoS One. 2014:9, e103351.

47. Mukaetova-Ladinska EB, Abdel-All Z, Andrade J, Alves da Silva J, O'Brien JT, Kalaria RN. Plasma and platelet clusterin ratio is altered in Alzheimer's disease patients with distinct neuropsychiatric symptoms: findings from a pilot study. Int J Geriatr Psychiatry. 2015;30:368-75.

48. Imhof A, Charnay Y, Vallet PG, Aronow B, Kovari E, French LE, et al. Sustained astrocytic clusterin expression improves remodeling after brain ischemia. Neurobiol Dis. 2006;22:274-83.

49. Ramakers $\mathrm{IH}$, Verhey FR, Scheltens $\mathrm{P}$, Hampel $\mathrm{H}$, Soininen $\mathrm{H}$, Aalten $\mathrm{P}$, et al. Anxiety is related to Alzheimer cerebrospinal fluid markers in subjects with mild cognitive impairment. Psychol Med. 2013;43:911-20.

50. Sokol DK, Maloney B, Long JM, Ray B, Lahiri DK. Autism, Alzheimer disease, and fragile X: APP, FMRP, and mGluR5 are molecular links. Neurology. 2011; 76:1344-52.

51. Craig D, Hart DJ, McCool K, Mcllroy SP, Passmore AP. Apolipoprotein E e4 allele influences aggressive behaviour in Alzheimer's disease. J Neurol Neurosurg Psychiatry. 2004;75:1327-30. 
52. van der Flier WM, Staekenborg S, Pijnenburg YA, Gillissen F, Romkes R, Kok $A$, et al. Apolipoprotein $E$ genotype influences presence and severity of delusions and aggressive behavior in Alzheimer disease. Dement Geriatr Cogn Disord. 2007:23:42-6.

53. Mehta PD, Pirttila T, Mehta SP, Sersen EA, Aisen PS, Wisniewski HM. Plasma and cerebrospinal fluid levels of amyloid beta proteins 1-40 and 1-42 in Alzheimer disease. Arch Neurol. 2000;57:100-5.

54. Lui JK, Laws SM, Li QX, Villemagne VL, Ames D, Brown B, et al. Plasma amyloid-beta as a biomarker in Alzheimer's disease: the AIBL study of aging. J Alzheimers Dis. 2010;20:1233-42.

55. Huang CW, Wang SJ, Wu SJ, Yang CC, Huang MW, Lin CH, et al. Potential blood biomarker for disease severity in the Taiwanese population with Alzheimer's disease. Am J Alzheimers Dis Other Demen. 2013;28:75-83.

56. Poljak A, Crawford JD, Smythe GA, Brodaty H, Slavin MJ, Kochan NA, et al. The relationship between plasma Abeta levels, cognitive function and brain volumetrics: Sydney Memory and Ageing Study. Curr Alzheimer Res. 2016; 13:243-55.

57. Figurski MJ, Waligorska T, Toledo J, Vanderstichele H, Korecka M, Lee VM, et al. Improved protocol for measurement of plasma beta-amyloid in longitudinal evaluation of Alzheimer's Disease Neuroimaging Initiative study patients. Alzheimers Dement. 2012;8:250-60.

58. Henriksen K, O'Bryant SE, Hampel H, Trojanowski JQ, Montine TJ, Jeromin A, et al. The future of blood-based biomarkers for Alzheimer's disease. Alzheimers Dement. 2014;10:115-31.

59. Janelidze S, Stomrud E, Palmqvist S, Zetterberg $H$, van Westen D, Jeromin A, et al. Plasma beta-amyloid in Alzheimer's disease and vascular disease. Sci Rep. 2016;6:26801.

60. Blasko I, Jungwirth S, Jellinger K, Kemmler G, Krampla W, Weissgram S, et al. Effects of medications on plasma amyloid beta (Abeta) 42: longitudinal data from the VITA cohort. J Psychiatr Res. 2008;:42:946-55.

61. Park JC, Han SH, Cho HJ, Byun MS, Yi D, Choe YM, et al. Chemically treated plasma Abeta is a potential blood-based biomarker for screening cerebral amyloid deposition. Alzheimers Res Ther. 2017;9:20

62. Brodaty H, Connors MH, Xu J, Woodward M, Ames D, PRIME study group. The course of neuropsychiatric symptoms in dementia: a 3-year longitudinal study. J Am Med Dir Assoc. 2015;16:380-7.

63. Metti AL, Cauley JA, Ayonayon HN, Harris TB, Rosano C, Williamson JD, et al. The demographic and medical correlates of plasma abeta 40 and abeta 42 . Alzheimer Dis Assoc Disord. 2013;27:244-9.

64. Mayeux R, Schupf N. Blood-based biomarkers for Alzheimer's disease: plasma Abeta40 and Abeta42, and genetic variants. Neurobiol Aging. 2011; 32 Suppl 1:S10-9.

65. Blennow K, Zetterberg H, Fagan AM. Fluid biomarkers in Alzheimer disease. Cold Spring Harb Perspect Med. 2012;2:a006221.

66. Hsu JL, Lee WJ, Liao YC, Lirng JF, Wang SJ, Fuh JL. Posterior atrophy and medial temporal atrophy scores are associated with different symptoms in patients with Alzheimer's disease and mild cognitive impairment. PLoS One. 2015;10, e0137121

\section{Submit your next manuscript to BioMed Central and we will help you at every step:}

- We accept pre-submission inquiries

- Our selector tool helps you to find the most relevant journal

- We provide round the clock customer support

- Convenient online submission

- Thorough peer review

- Inclusion in PubMed and all major indexing services

- Maximum visibility for your research

Submit your manuscript at www.biomedcentral.com/submit 